\title{
INFLUENCE OF OPTICAL FILTERS ON THE PERFORMANCE OF FSK/IM TRANSMISSION SCHEME
}

\author{
J. J. Vegas Olmos ${ }^{1}$, I. Tafur Monroy ${ }^{1}$, E. Tangdiongga ${ }^{1}$, J.P.A. van Berkel ${ }^{1}$, A.M.J. \\ Koonen ${ }^{1}$ and J. Prat ${ }^{2}$ \\ ${ }^{I}$ COBRA Research Institute, Eindhoven University of Technology, The Netherlands. E-mail \\ j.j.vegas@tue.nl \\ ${ }^{2}$ GCO Optical Communications Group, Technical University of Catalonia, Spain
}

\begin{abstract}
Influence of the filter shape on the performance of a single-wavelength combined FSK/IM scheme is investigated by simulations and experiments. For $156 \mathrm{Mbit} / \mathrm{s}$ FSK label and $10 \mathrm{Gbit} / \mathrm{s}$ IM payload, central wavelength misalignment of the signal and the optical filter can be tolerated up to 30 $\mathrm{GHz}$ without noticeable penalties. Results of the simulation agree very well with the experiments.
\end{abstract}

\section{INTRODUCTION}

All-optical label switching (OLS) is a promising technique for switching internet protocol (IP) packet and for forwarding optical functions over Wavelength Division Multiplexing (WDM) networks [1]. By using short fixed-length labels the core nodes of the network can forward/switch packets quickly and efficiently while keeping the payload data entirely in the optical domain. A combined frequency-shift keying/intensity modulation (FSK/IM) scheme is a strong candidate for such an optical data router because of the simple label swapping mechanism and the scalability to higher data rates [2]. In this orthogonal modulation scheme, the payload is intensity modulated and the label is frequency modulated. When reaching an optical node, the label is separated from the payload 
and is subsequently processed. A new label will be created and re-inserted together with the payload to form a complete data packet. During the label processing, the payload remains unchanged in the optical domain. Recently, the generation of an FSK signal by using an agile tuneable laser is reported in [3]. A 10 Gbit/s FSK signal generated by a phase modulator is demonstrated in [4].

When an optical network is considered which consists of a number of optical nodes, one of the main network impairments is the spectral misalignment between the central wavelength of the modulated signal and the wavelength selective element of the nodes, such as arrayed-waveguide (AWG)-based optical filters $[5,6]$. This spectral misalignment will inevitably increase the network susceptibility to power loss and optical crosstalk in case of WDM transmission.

This destructive effect becomes stronger in the case of the combined FSK/IM scheme, where the broadening of the spectrum due to FSK modulation will not only disturb the signal-filter alignment but it can also shift a portion of the signal spectrum outside the filter bandwidth, possibly leading to a serious signal deformation.

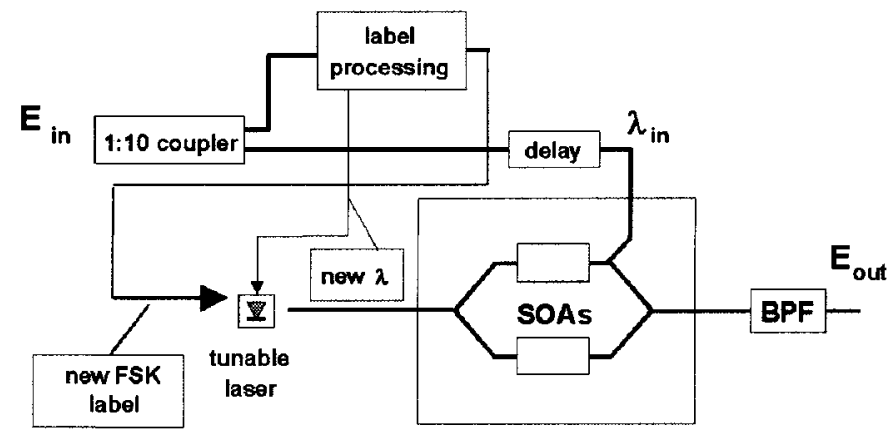

Figure 1. Generic node architecture for all optical packet switched. BPF: Band pass filter

Figure 1 shows a generic node structure and a label swapper architecture of the combined FSK/IM scheme. Ideally, the signal passes through an optical filter, for instance an optical (de)multiplexer or an optical (tuneable) band pass filter. This filter is in general needed in optically amplified WDM systems for selecting a desired wavelength channel and removing the outband amplified spontaneous emission (ASE) noise. To cope with signal-filter misalignment in an optical network, it is necessary to give extra margin in the power budget allocation. The FSK label with a sufficient optical power is locally generated in each node. Consequently, the critical constrain comes from the IM payload signal which is 
generated from a distant node and is kept in the optical domain in the nodes. We present in this paper a theoretical and experimental study on the performance of the combined FSK/M scheme impaired by the filter shape.

\subsection{Simulations}

A continuous wave (CW) laser source, a chirp-free IM modulator, a FSK modulator, and a third order Gaussian optical bandpass filter (BPF) are assumed in the simulation. Both modulators are set to be lossless. The data format of the IM payload and FSK label signal is $2^{7}-1$ pseudorandom nonreturn-to-zero (NRZ). The payload signal is coded at $10 \mathrm{Gbit} / \mathrm{s}$ and the label signal $156.25 \mathrm{Mbit} / \mathrm{s}$. The label bitrate is set to be a subrate of the payload bitrate. The FSK labeling of the IM payload gives rise to a frequency deviation of $15 \mathrm{GHz}$. The optical receiver is modeled as a normal photodiode followed by a Bessel third order electrical low pass filter with a full-width half maximum (FWHM) bandwidth of 0.7 times the payload bit rate. The $\mathrm{CW}$ laser center frequency is set to $192.3 \mathrm{THz}$ and the BPF center frequency is swept from $192.30 \mathrm{THz}$ to $192.36 \mathrm{THz}$. Error probability is calculated by using the Gaussian tail integration method. The detection threshold is fixed at the halfway of mark and space. The detection penalty corresponds to the input power for which the error probability equals $10^{-9}$. Figure 2 shows the penalty as a function of frequency detuning for different FHWM values of the optical BPF.

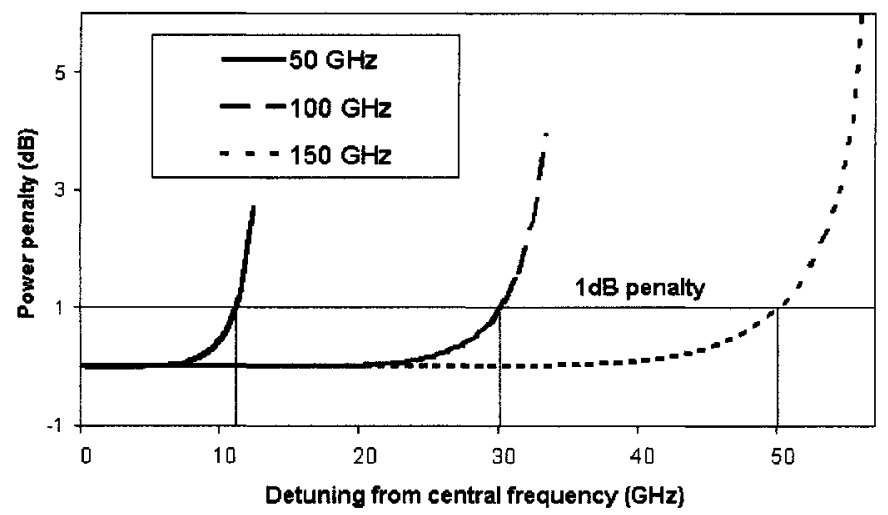

Figure 2. Penalty of the IM signal for the combined FSK/IM signal versus the detuning from the center wavelength of filters with different bandwidths 
We take a penalty of $1 \mathrm{~dB}$ as the reference for performance evaluation. An optical filter with $50 \mathrm{GHz}$ bandwidth is more sensitive to the frequency detuning than filters with larger bandwidths. For 1-dB penalty we can observe that the tolerance to the frequency misalignment increases with approximately $20 \mathrm{GHz}$ as the bandwidth is enhanced with $50 \mathrm{GHz}$. The signal-filter frequency detuning must be kept under $12 \mathrm{GHz}$ for a $50 \mathrm{GHz}$ optical filter with penalties under $1 \mathrm{~dB}$.

\section{EXPERIMENTAL SETUP}

FSK modulation on the signal is obtained by modulating the current to the phase section of a tuneable laser source of type single grating assisted coupler sampled reflector (GCSR) [3]. The label format is again pseudorandom $2^{7}-1 \mathrm{NRZ}$ at $100 \mathrm{Mbit} / \mathrm{s}$ bit-rate. The current to the other laser sections (coupler, reflector, and gain) is used for coarse and fine-tuning to a target wavelength [7], which is in the range of $1529.551561 .42 \mathrm{~nm}$. For this experiment the laser wavelength is 1558.92 $\mathrm{nm}$ or $192.30 \mathrm{THz}$ with a frequency deviation of $15 \mathrm{GHz}$ due to the FSK modulation format. The experimental setup is schematically depicted in Fig. 3.

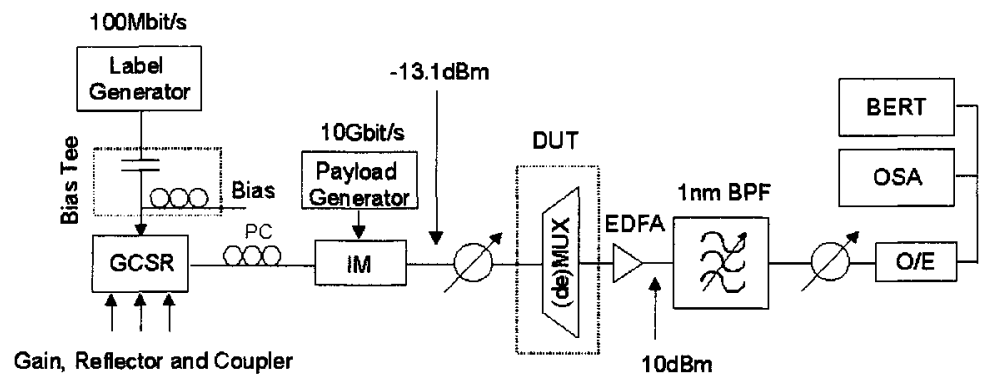

Figure 3. Experimental setup. DUT: Device under test. BPF: Band pass filter

After the labeling section, the signal is intensity modulated by using a MachZehnder amplitude modulator with the same data format as the label but at 10 Gbit/s bit-rate. The combined FSK/IM signal has an extinction ratio of $6.2 \mathrm{~dB}$ and a $Q$ factor of 8.5. This signal is launched to the device under test (DUT), which is an 8-channel AWG (de)multiplexer. Figure 4 shows the transmittance of the channel we used in the experimental assessment. The spectral response can be sufficiently modelled as a first order Gaussian filter with a FWHM bandwidth of $100 \mathrm{GHz}$. 


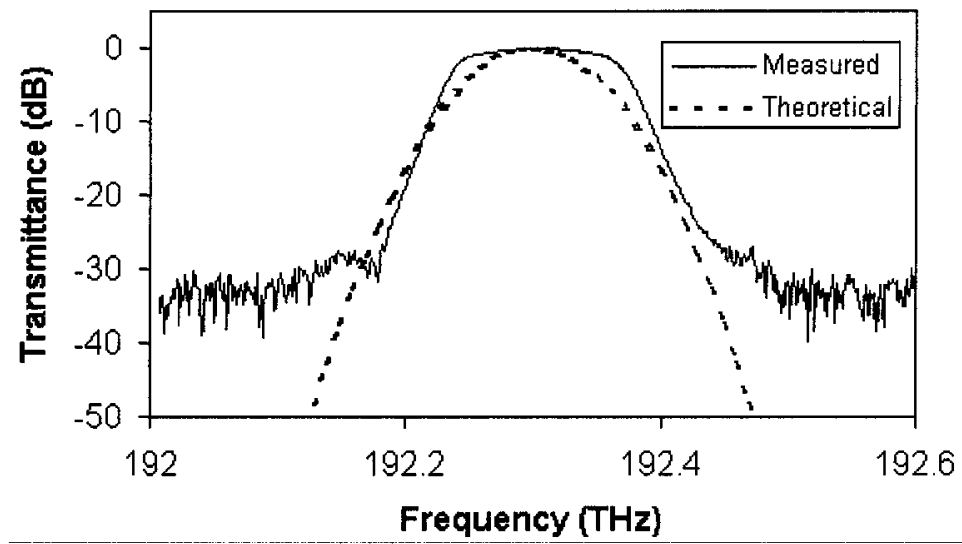

Figure 4. Theoretical and measured filter profile of $100-\mathrm{GHz}$ bandwidth at $192.3 \mathrm{THz}$

Although the measured filter center frequency deviates $10 \mathrm{GHz}$ from the theoretical one, the passband shape looks fairly identical. The laser frequency is tuned by changing the laser temperature with a step of $0.3^{\circ} \mathrm{C}$. This step corresponds to a frequency step of $1 \mathrm{GHz}$. Due to the symmetrical spectral response of the filter around the center wavelength and the symmetrical FSK/IM spectral response to the pseudorandom bit patterns, only the performance degradation by a positive frequency detuning is evaluated. A negative frequency detuning gives a similar result. 


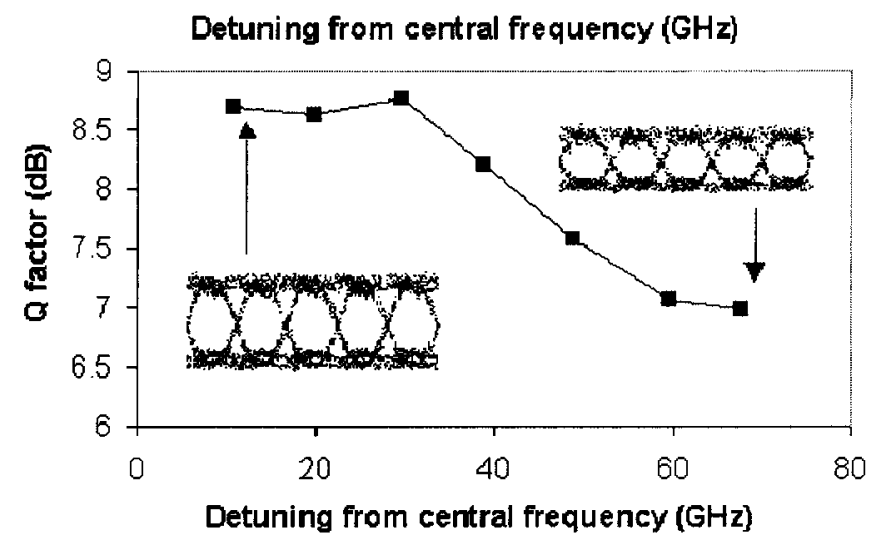

Figure 5. Performance of filtered FSK/IM signal as a function of frequency detuning: (a) received optical spectra and (b) $Q$ value

Figure 5 shows the $Q$ factor when the laser center frequency is swept within the filter bandwidth. For clarity, we displayed eye patterns at two extreme detuning frequencies. As observed in Figure 5, the $\mathrm{Q}$ factor is preserved for the detuning frequencies until $30 \mathrm{GHz}$ and drops considerably by almost $2 \mathrm{~dB}$ at $70-\mathrm{GHz}$ detuning. This is caused by the fact that one of the FSK peaks is converted into amplitude fluctuation, which in turn impairs the IM payload signal. The experimental values are confirmed by the simulations.

\section{CONCLUSION}

We have presented a comprehensive study on the impact of the filter shape on the performance of the combined scheme FSK/IM in a single channel for several filter bandwidths. From simulations and experiments, we observed that the frequency misalignments up to $30 \mathrm{GHz}$ of the signal and the Gaussian filter can be accepted without noticeable degradation of the IM signal in systems operating at $10 \mathrm{Gbit} / \mathrm{s}$ payload. For OBS networks where the signal is regenerated locally at every node by the wavelength conversion, no significant performance degradation is expected. 


\section{ACKNOWLEDGMENTS}

The European Commission is acknowledged for partially funding this work within the IST project STOLAS (Switching Technologies for Optically Labeled Signals).

\section{REFERENCES}

[1] Chunming Qiao, "Labeled Optical Burst Switching for IP-over-WDM Integration", IEEE Communication Magazine, September 2000, pp.104-114.

[2] Ton Koonen, Geert Morthier, Jean Jennen, Huug de Waardt, Piet Demeester, "Optical packet routing in IP-over-WDM networks deploying two-level optical labeling", Proc. of ECOC'01, Amsterdam, Sep. 30 - Oct. 4, 2001, paper Th.L.2.1, pp. 608-609.

[3] J.J. Vegas Olmos, I. Tafur Monroy and A.M.J. Koonen, "High bit-rate combined FSK/IM modulated optical signal generation by using GSCR tunable laser sources," Optics Express, Vol. 11, 3136-3140 (2003).

[4] Tetsuya Kawanishi, Takahide Sakamoto, Satoshi Shinada, Masayuki Izutsu, Kaoru Higuma, Takahisa Fujita, Junichiro Ichikawa, "High-speed optical FSK modulator for optical packet labeling," Postdeadline paper in OFC 2004, Paper PDP16, Los Angeles, 2004.

[5] N. Khrais, A. Elrefaie, R. Wagner and S. Ahmed, "Performance degradation of multiwavelength optical networks due to laser and (de)multiplexer misalignments," IEEE Photonic Technology Letters, vol. 7, pp. 1348-1350, Nov. 1995.

[6] C. Caspar, H. Foisel, R. Freund, U. Kruger and B. Strebel, "Cascadability of arrayedwaveguide grating (de)multiplexers in transparent optical networks," Proc. of OFC'97, 1997, Paper TUE2, pp.19-20.

[7] O. Lavrova and D.J.Blumenthal, "Detailed transfer matrix based dynamic model for multisection widely-tunable GSCR lasers," J. Lightwave Technol., vol. 18, pp. 12741283 , Sept. 2000. 